\title{
Achieving transparency and maximizing scattering with metamaterial-coated conducting cylinders
}

\author{
Erdinc Irci and Vakur B. Ertürk \\ Department of Electrical and Electronics Engineering, Bilkent University, 06800 Bilkent, Ankara, Turkey
}

(Received 8 June 2007; revised manuscript received 14 August 2007; published 9 November 2007)

\begin{abstract}
In this work, the electromagnetic interaction of plane waves with infinitely long metamaterial-coated conducting cylinders is considered. Different from "conjugate" pairing of double-positive (DPS) and doublenegative (DNG) or epsilon-negative (ENG) and mu-negative (MNG) concentric cylinders, achieving transparency and maximizing scattering are separately achieved by covering perfect electric conductor (PEC) cylinders with simple (i.e., homogeneous, isotropic, and linear) metamaterial coatings. The appropriate constitutive parameters of such metamaterials are investigated for Transverse Magnetic (TM) and in particular for Transverse Electric (TE) polarizations. For TE polarization it is found out that the metamaterial-coating permittivity has to be in the $0<\varepsilon_{c}<\varepsilon_{0}$ interval to achieve transparency, and in the $-\varepsilon_{0}<\varepsilon_{c}<0$ interval to achieve scattering maximization. However, unlike the "conjugate" pairing of DPS-DNG or ENG-MNG cases, when the transparency for metamaterial-coated PEC cylinders are considered, the analytically found relation between $\varepsilon_{c}$ and the ratio of core-coating radii, $\gamma$, should be modified in a sense that scattering from the PEC core is canceled by the coating. Furthermore, replacing $\varepsilon$ by $\mu$ (and vice versa) does not lead to the same conclusions for TM polarization unless the PEC cylinder is replaced by a perfect magnetic conductor (PMC) cylinder. On the other hand, scattering maximization can also be achieved in the TM polarization case when coating permeability $\mu_{c}<0$, whereas transparency requires large $\left|\mu_{c}\right|$ for this polarization. Numerical results in the form of normalized monostatic and bistatic echo widths, which demonstrate the transparency and scattering maximization phenomena, are given and possible application areas are discussed.
\end{abstract}

DOI: 10.1103/PhysRevE.76.056603

PACS number(s): 42.70.-a, 42.79.-e

\section{INTRODUCTION}

With their peculiar and distinctive electromagnetic properties, metamaterials have gained an increasing interest among the scientific community in the recent years. Although the theoretical background was established long before $[1-3]$, the feasibility remained as a question mark until the experimental verification [4]. The flexibility they have brought with their exceptional properties like negative refraction, negative permittivity and/or negative permeability, give rise to possible utilizations of metamaterials in different scientific and engineering applications, which otherwise cannot be easily accomplished with conventional materials. Recently, reducing scattering from various structures, and in the limit achieving transparency and building cloaking structures, have been investigated by many researchers [5-11]. On the other hand, resonant structures aimed at increasing the electromagnetic intensities, stored or radiated power levels have also been studied extensively [10,12-18]. Similarly, metamaterial layers have been proposed to enhance the power radiated by electrically small antennas [19-21].

As expected, reducing the radar cross sections (RCS) of aircrafts and missiles is very crucial for military applications. Achieving transparency with cloaking structures is an ultimate goal. Ideally, transparency means full transmission of the incident wave in the direction of incidence with no scattering in any other direction. In this work, by transparency we particularly mean the significant reduction of scattering in the backscattering direction. On the other hand, RCS maximization of very tiny structures is suitable for radar antimeasures (e.g., chaff) or as inclusions in host bodies as resonators. The perfect electric conductor (PEC) core cylin- ders we investigate here may also ease the coating process, especially when plasmonic covers utilizing surface plasmons are used [22].

The transparency and resonance (scattering maximization) conditions investigated in $[9,10,12-16]$ are mainly attributed to pairing of "conjugate" materials: materials which have opposite signs of constitutive parameters [e.g., doublepositive (DPS) and double-negative (DNG) or epsilonnegative (ENG) and mu-negative (MNG)]. In [9], electrically small dielectric spheres are covered with metamaterial coatings to achieve transparency. Although no numerical result is provided, transparency conditions for its cylindrical counterpart (again dielectric small cylinders coated with metamaterial coatings) as well as for impenetrable spheres, as a limiting case, are briefly mentioned. More recently, independent from the work presented here, covering impenetrable spheres with metamaterial coatings to achieve transparency is presented in [11]. The opposite resonance effect, which enhances the scattering dramatically for tiny sub-wavelength dielectric spheres, is presented in [15]. A more detailed study of cylindrical geometries was previously done in [12].

Considering the fact that many applications (e.g., airborne targets) are usually in cylindrical shape and they are treated as PEC in electromagnetic (EM) solvers, in the present work we extend the results of $[9,12]$ to achieve transparency and resonance for cylindrical structures when the core cylinder is particularly PEC by using simple (i.e., homogeneous, isotropic, and linear) metamaterial or plasmonic coatings. As in the case of "conjugate" pairing [9,12-15], transparency and resonance are found to be dependent on the ratio of corecoating radii. However, the presence of PEC core (instead of a penetrable core) requires a different ratio of core-coating 
radii expression than the one presented in [9]. In [9], this expression (i.e., ratio of core-coating radii) is derived based on eliminating the dipolar terms for small spheres. However, in this work, we use the dipolar terms to cancel the scattering from the core PEC cylinder, which is small. Thus, existence of these terms is essential. Furthermore, as the electrical size of the core PEC cylinder increases, in addition to the dipolar terms, higher order terms should be incorporated to cancel the scattering from the PEC core. Consequently, in our work we show that, for Transverse Electric (TE) polarization, the metamaterial coating should have $0<\varepsilon_{c}<\varepsilon_{0}$ as its permittivity to achieve transparency, whereas the coating permittivity has to be in the $-\varepsilon_{0}<\varepsilon_{c}<0$ interval for resonance so that scattering maximization can be achieved.

Besides, notice that because the core cylinder is PEC, unlike the aforementioned "conjugate" pairing cases $[9,15]$, the analytical relations we have derived for TE polarization cannot be used for Transverse Magnetic (TM) polarization by interchanging $\varepsilon$ with $\mu$ (and vice versa), unless the core cylinder is replaced with perfect magnetic conductor (PMC). Yet both transparency and resonant peaks can be achieved for TM polarization. Here, we show numerically that for electrically small PEC cylinders transparency can be obtained by covering them with metamaterial covers having large $\left|\mu_{c}\right|$, whereas resonant peaks are observed when $\mu_{c}$ $<0$.

The organization of this paper is as follows. In Sec. II, the geometry of the problem and the theoretical background are given. Conditions for both transparency and resonance (scattering maximization) are provided in Secs. III and IV, respectively. Section V is composed of numerical results, mainly in the form of monostatic and bistatic echo widths, to validate the transparency and resonance conditions as well as their discussions. In this work, as a measure of scattering, we use the RCS definition and we imply the 2D normalized monostatic or bistatic echo widths (i.e., $\sigma / \lambda_{0} ; \lambda_{0}$ is the free space wavelength). An $e^{j \omega t}$ time dependence is assumed and suppressed throughout this paper.

\section{THEORETICAL BACKGROUND}

Consider a PEC cylinder of infinite length, having radius $a$, which is covered by a concentric metamaterial coating with outer radius $b>a$. The metamaterial coating is assumed to be homogeneous, isotropic, and linear, thus a simple material, having permittivity $\varepsilon_{c}$ and permeability $\mu_{c}$, and surrounded by free space $\left(\varepsilon_{0}, \mu_{0}\right)$. The geometry of the problem is depicted in Fig. 1.

The metamaterial-coated PEC cylinder is illuminated normally by a uniform plane wave which travels in the direction that makes an angle $\phi_{0}$ with the $+x$ axis. The scattering and transmission by the metamaterial coated PEC cylinder is investigated for the cases where the polarization of the plane wave is either $\mathrm{TM}^{z}$ or $\mathrm{TE}^{z}$.

\section{A. $\mathbf{T M}^{z}$ polarization}

For the $\mathrm{TM}^{z}$ polarized uniform plane wave, referring to Fig. 1 the incident electric field can be written as

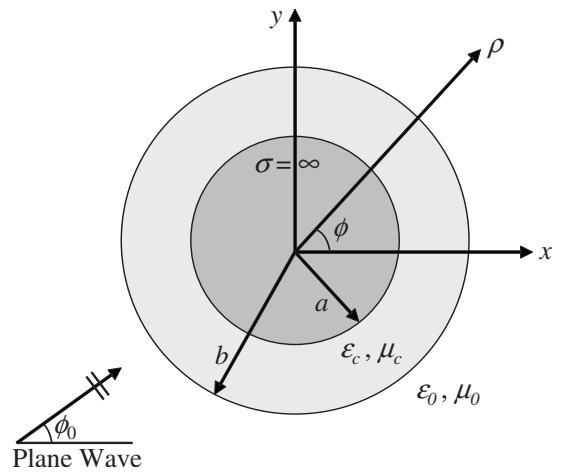

FIG. 1. Cross section of a PEC cylinder of infinite length covered by a concentric metamaterial coating.

$$
E_{z}^{i}=E_{0} e^{-j k_{0}\left(x \cos \phi_{0}+y \sin \phi_{0}\right)}=E_{0} e^{-j k_{0} \rho \cos \left(\phi-\phi_{0}\right)},
$$

where $x=\rho \cos \phi, y=\rho \sin \phi$, and $k_{0}=\omega \sqrt{\mu_{0} \varepsilon_{0}}$ is the free space wave number.

Utilizing a similar procedure as in [23], the incident, transmitted and scattered electric fields can be represented respectively as

$$
\begin{gathered}
E_{z}^{i}=E_{0} \sum_{n=-\infty}^{+\infty} j^{-n} J_{n}\left(k_{0} \rho\right) e^{j n\left(\phi-\phi_{0}\right)}, \quad \rho \geqslant b, \\
E_{z}^{t}=E_{0} \sum_{n=-\infty}^{+\infty} j^{-n}\left[a_{n}^{\mathrm{TM}} J_{n}\left(k_{c} \rho\right)+b_{n}^{\mathrm{TM}} Y_{n}\left(k_{c} \rho\right)\right] e^{j n\left(\phi-\phi_{0}\right)}, \\
a \leqslant \rho \leqslant b, \\
E_{z}^{s}=E_{0} \sum_{n=-\infty}^{+\infty} j^{-n} c_{n}^{\mathrm{TM}} H_{n}^{(2)}\left(k_{0} \rho\right) e^{j n\left(\phi-\phi_{0}\right)}, \quad \rho \geqslant b,
\end{gathered}
$$

where $k_{c}=\omega \sqrt{\mu_{c} \varepsilon_{c}}$ is the wave number in the metamaterial coating. $a_{n}^{\mathrm{TM}}, b_{n}^{\mathrm{TM}}$, and $c_{n}^{\mathrm{TM}}$ are the unknown coefficients which are to be determined from the boundary conditions. At the interface between the PEC cylinder and the metamaterial coating, tangential component of the electric field (i.e., $E_{z}$ ) should be zero. On the outer boundary of the metamaterial coating, tangential components of the electric and magnetic fields (i.e., $E_{z}$ and $H_{\phi}$, respectively) should be continuous. The simultaneous solution of these boundary conditions can be written in a matrix-vector product form and the unknown coefficients can be found from

$$
\left[\begin{array}{c}
a_{n}^{\mathrm{TM}} \\
b_{n}^{\mathrm{TM}} \\
c_{n}^{\mathrm{TM}}
\end{array}\right]=\left[\begin{array}{ccc}
J_{n}\left(k_{c} a\right) & Y_{n}\left(k_{c} a\right) & 0 \\
J_{n}\left(k_{c} b\right) & Y_{n}\left(k_{c} b\right) & -H_{n}^{(2)}\left(k_{0} b\right) \\
J_{n}^{\prime}\left(k_{c} b\right) & Y_{n}^{\prime}\left(k_{c} b\right) & -\zeta H_{n}^{(2)^{\prime}}\left(k_{0} b\right)
\end{array}\right]^{-1}\left[\begin{array}{c}
0 \\
J_{n}\left(k_{0} b\right) \\
\zeta J_{n}^{\prime}\left(k_{0} b\right)
\end{array}\right],
$$

where $\zeta=\eta_{c} / \eta_{0} . \eta_{c}=\sqrt{\mu_{c} / \varepsilon_{c}}$ and $\eta_{0}=\sqrt{\mu_{0} / \varepsilon_{0}}$ are the wave impedances of the metamaterial coating and free space, respectively. The derivatives of the Bessel and Hankel functions in Eq. (5) are taken with respect to their entire arguments. 
Far field expression for the scattered electric field is obtained using the large argument approximation of Hankel functions. Normalized bistatic echo width is then found as

$$
\sigma^{\mathrm{TM}} / \lambda_{0}=\lim _{\rho \rightarrow \infty}\left[2 \pi \rho \frac{\left|E_{z}^{s}\right|^{2}}{\left|E_{z}^{i}\right|^{2}}\right] / \lambda_{0}=\frac{2}{\pi}\left|\sum_{n=-\infty}^{+\infty} c_{n}^{\mathrm{TM}} e^{j n\left(\phi-\phi_{0}\right)}\right|^{2} .
$$

\section{B. TE $^{z}$ polarization}

In the $\mathrm{TE}^{z}$ polarization case, the incident, transmitted and scattered magnetic fields can be written respectively as

$$
\begin{gathered}
H_{z}^{i}=H_{0} \sum_{n=-\infty}^{+\infty} j^{-n} J_{n}\left(k_{0} \rho\right) e^{j n\left(\phi-\phi_{0}\right)}, \quad \rho \geqslant b, \\
H_{z}^{t}=H_{0} \sum_{n=-\infty}^{+\infty} j^{-n}\left[a_{n}^{\mathrm{TE}} J_{n}\left(k_{c} \rho\right)+b_{n}^{\mathrm{TE}} Y_{n}\left(k_{c} \rho\right)\right] e^{j n\left(\phi-\phi_{0}\right)},
\end{gathered}
$$

$$
a \leqslant \rho \leqslant b
$$

$$
H_{z}^{s}=H_{0} \sum_{n=-\infty}^{+\infty} j^{-n} c_{n}^{\mathrm{TE}} H_{n}^{(2)}\left(k_{0} \rho\right) e^{j n\left(\phi-\phi_{0}\right)}, \quad \rho \geqslant b .
$$

Utilizing similar boundary conditions to the $\mathrm{TM}^{z}$ polarization, the following system of equations is obtained:

$$
\left[\begin{array}{c}
a_{n}^{\mathrm{TE}} \\
b_{n}^{\mathrm{TE}} \\
c_{n}^{\mathrm{TE}}
\end{array}\right]=\left[\begin{array}{ccc}
J_{n}^{\prime}\left(k_{c} a\right) & Y_{n}^{\prime}\left(k_{c} a\right) & 0 \\
J_{n}\left(k_{c} b\right) & Y_{n}\left(k_{c} b\right) & -H_{n}^{(2)}\left(k_{0} b\right) \\
\zeta J_{n}^{\prime}\left(k_{c} b\right) & \zeta Y_{n}^{\prime}\left(k_{c} b\right) & -H_{n}^{(2)^{\prime}}\left(k_{0} b\right)
\end{array}\right]^{-1}\left[\begin{array}{c}
0 \\
J_{n}\left(k_{0} b\right) \\
J_{n}^{\prime}\left(k_{0} b\right)
\end{array}\right] .
$$

The scattering coefficient, $c_{n}^{\mathrm{TE}}$, can be found from Eq. (10) as

$$
c_{n}^{\mathrm{TE}}=\frac{\zeta J_{n}\left(k_{0} b\right)\left[J_{n}^{\prime}\left(k_{c} a\right) Y_{n}^{\prime}\left(k_{c} b\right)-J_{n}^{\prime}\left(k_{c} b\right) Y_{n}^{\prime}\left(k_{c} a\right)\right]-J_{n}^{\prime}\left(k_{0} b\right)\left[J_{n}^{\prime}\left(k_{c} a\right) Y_{n}\left(k_{c} b\right)-J_{n}\left(k_{c} b\right) Y_{n}^{\prime}\left(k_{c} a\right)\right]}{H_{n}^{(2)}\left(k_{0} b\right)\left[J_{n}^{\prime}\left(k_{c} a\right) Y_{n}\left(k_{c} b\right)-J_{n}\left(k_{c} b\right) Y_{n}^{\prime}\left(k_{c} a\right)\right]-\zeta H_{n}^{(2)}\left(k_{0} b\right)\left[J_{n}^{\prime}\left(k_{c} a\right) Y_{n}^{\prime}\left(k_{c} b\right)-J_{n}^{\prime}\left(k_{c} b\right) Y_{n}^{\prime}\left(k_{c} a\right)\right]} .
$$

Normalized bistatic echo width $\sigma^{\mathrm{TE}} / \lambda_{0}$ is the same as Eq. (6), except electric fields are replaced by magnetic fields.

\section{Complex analysis of the wave number and the wave impedance of the metamaterial coating}

In accordance with the Drude and Lorentz medium models in [24-26], the metamaterial coating is assumed to have a small loss near its plasma frequency. Therefore, in the theoretical analysis, constitutive parameters of the metamaterial coating are selected as complex quantities. Consequently, the wave number and the wave impedance of the metamaterial coating are also complex quantities.

The permittivity and the permeability of the metamaterial coating can be expressed in polar form, respectively, as

$$
\varepsilon_{c}=\left|\varepsilon_{c}\right| e^{j \phi_{\varepsilon_{c}}}, \quad \mu_{c}=\left|\mu_{c}\right| e^{j \phi_{\mu_{c}}} .
$$

Similarly, the wave number and the wave impedance of the metamaterial coating can be written as

$$
k_{c}=\omega \sqrt{\mu_{c} \varepsilon_{c}}=\left|k_{c}\right| e^{j \phi_{k_{c}}}, \quad \eta_{c}=\sqrt{\mu_{c} / \varepsilon_{c}}=\left|\eta_{c}\right| e^{j \phi_{\eta_{c}}}
$$

respectively, where

$$
\left|k_{c}\right|=\omega \sqrt{\left|\mu_{c}\right|\left|\varepsilon_{c}\right|}, \quad\left|\eta_{c}\right|=\sqrt{\left|\mu_{c}\right| /\left|\varepsilon_{c}\right|},
$$

with

$$
\phi_{k_{c}}=\frac{1}{2}\left(\phi_{\mu_{c}}+\phi_{\varepsilon_{c}}\right), \quad \phi_{\eta_{c}}=\frac{1}{2}\left(\phi_{\mu_{c}}-\phi_{\varepsilon_{c}}\right) .
$$

The choice of branches for the square roots in Eq. (15) is based on causality in a linear dispersive medium, the wave directions associated with reflection and transmission from the interfaces and the direction of electromagnetic power

\begin{tabular}{|c|c|c|c|c|}
\hline & $\phi_{\mu_{c}}$ & $\phi_{\varepsilon_{c}}$ & $\phi_{k_{c}}$ & $\phi_{\eta_{c}}$ \\
\hline DPS & $\left(-\frac{\pi}{2}, 0\right.$ & $\left(-\frac{\pi}{2}, 0\right.$ & $\left(-\frac{\pi}{2}, 0\right]$ & $\left(-\frac{\pi}{4}, \frac{\pi}{4}\right)$ \\
\hline DNG & $-\pi,-\frac{\pi}{2}$ & $-\pi,-\frac{\pi}{2}$ & {$\left[-\pi,-\frac{\pi}{2}\right)$} & $\left(-\frac{\pi}{4}, \frac{\pi}{4}\right)$ \\
\hline MNG & $-\pi,-\frac{\pi}{2}$ & $\left(-\frac{\pi}{2}, 0\right.$ & $\left.-\frac{3 \pi}{4},-\frac{\pi}{4}\right)$ & {$\left[-\frac{\pi}{2}, 0\right)$} \\
\hline ENG & $\left(-\frac{\pi}{2}, 0\right.$ & $-\pi,-\frac{\pi}{2}$ & $\left.-\frac{3 \pi}{4},-\frac{\pi}{4}\right)$ & $\left(0, \frac{\pi}{2}\right]$ \\
\hline
\end{tabular}
flow. This choice, which was given and examined in details in [26] for DNG metamaterials, is also used for DPS, MNG, and ENG metamaterials. The arguments of $\mu_{c}, \varepsilon_{c}, k_{c}$ and $\eta_{c}$ for these metamaterials are tabulated in Table I.

Examination of Table I shows that for lossless DPS medium, the wave number is real and positive. For lossless DNG medium, the wave number is real and negative. For lossless MNG and ENG media, the wave number is negative

TABLE I. Arguments of $\mu_{c}, \varepsilon_{c}, k_{c}$, and $\eta_{c}$ for different media. 
and imaginary, which shows the presence of evanescent waves.

In majority of the numerical experiments, we have investigated the lossless cases for convenience. Therefore, if not stated otherwise, the metamaterial coating should be treated lossless in the numerical experiments.

\section{TRANSPARENCY CONDITION}

The transparency condition for $\mathrm{TE}^{z}$ polarization is derived by setting the numerator of the scattering coefficient $c_{n}^{\mathrm{TE}}$ given in Eq. (11) to zero. In the subwavelength limit, assuming $\left|k_{c}\right| a<\left|k_{c}\right| b \ll 1, k_{0} b \ll 1$ and utilizing the small argument forms of Bessel and Hankel functions, the following transparency condition is obtained:

$$
\gamma=\sqrt[2 n]{\frac{\varepsilon_{0}-\varepsilon_{c}}{\varepsilon_{0}+\varepsilon_{c}}} \text { for } n \neq 0,
$$

where $\gamma=a / b$ is the ratio of core-coating radii and $n$ is the index of series summation.

Alternatively, one can use the transparency condition for an electrically small cylindrical scatterer, which is composed of two concentric layers of different isotropic materials, given in [9] for the $\mathrm{TE}^{z}$ polarization as

$$
\begin{gathered}
\gamma=\sqrt[2 n]{\frac{\left(\varepsilon_{c}-\varepsilon_{0}\right)\left(\varepsilon_{c}+\varepsilon\right)}{\left(\varepsilon_{c}-\varepsilon\right)\left(\varepsilon_{c}+\varepsilon_{0}\right)}} \text { for } n \neq 0, \\
\gamma=\sqrt{\frac{\mu_{c}-\mu_{0}}{\mu_{c}-\mu}} \text { for } n=0,
\end{gathered}
$$

where $(\varepsilon, \mu)$ are constitutive parameters of the core cylinder and $\left(\varepsilon_{c}, \mu_{c}\right)$ are constitutive parameters of the coating (shell) layer.

When the core cylinder is PEC, $\varepsilon \rightarrow-j \infty$ and $\mu=\mu_{0}$. In this case Eq. (18) becomes

$$
\gamma=\sqrt{\frac{\mu_{c}-\mu_{0}}{\mu_{c}-\mu_{0}}}=1 \quad \text { for } \mu_{c} \neq \mu_{0}, \quad n=0,
$$

which means there would be no coating. However, Eq. (17) can still be used in the limiting case, yielding the same transparency condition in Eq. (16) as

$$
\gamma \rightarrow \sqrt[2 n]{\frac{\left(\varepsilon_{c}-\varepsilon_{0}\right)\left(\varepsilon_{c}-j \infty\right)}{\left(\varepsilon_{c}+j \infty\right)\left(\varepsilon_{c}+\varepsilon_{0}\right)}}=\sqrt[2 n]{\frac{\varepsilon_{0}-\varepsilon_{c}}{\varepsilon_{0}+\varepsilon_{c}}} \text { for } n \neq 0 .
$$

The root in Eq. (16) is of even degree of $n$ (i.e., $2 n$ ), which implies that the argument of the root must be positive. On the other hand, when there is a coating $\gamma$ should vary between 0 and 1 . Therefore,

$$
0<\frac{\varepsilon_{0}-\varepsilon_{c}}{\varepsilon_{0}+\varepsilon_{c}}<1,
$$

which leads to

$$
0<\frac{\varepsilon_{0}-\varepsilon_{c}}{\varepsilon_{0}+\varepsilon_{c}} \Rightarrow-\varepsilon_{0}<\varepsilon_{c}<\varepsilon_{0}
$$

and

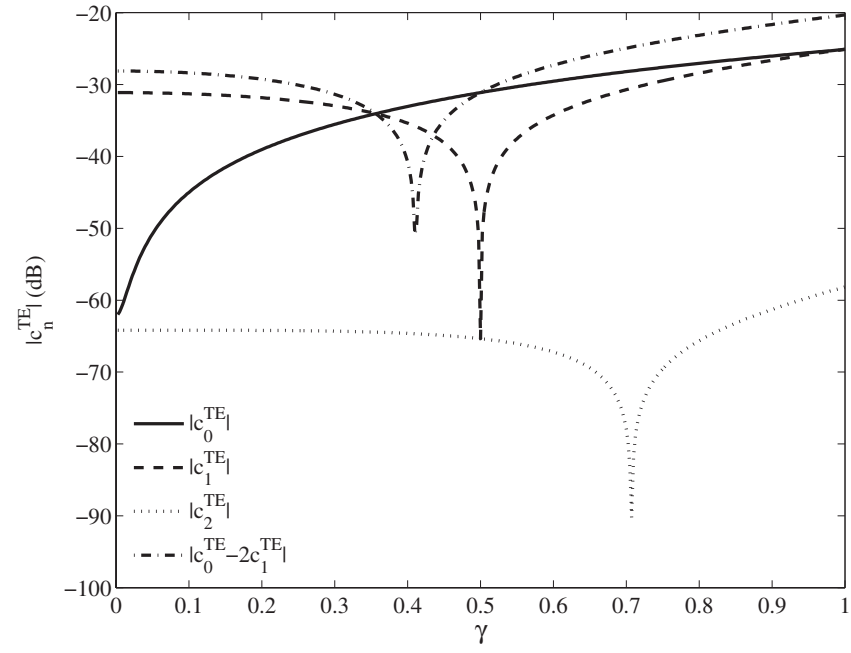

FIG. 2. Magnitudes of several scattering coefficients $\left(\varepsilon_{c}=0.6 \varepsilon_{0}, \mu_{c}=\mu_{0}\right.$, and $\left.b=\lambda_{0} / 100\right)$.

$$
\frac{\varepsilon_{0}-\varepsilon_{c}}{\varepsilon_{0}+\varepsilon_{c}}<1 \Rightarrow \varepsilon_{c}<-\varepsilon_{0} \text { or } 0<\varepsilon_{c} .
$$

From Eqs. (22) and (23), the proper choice for $\varepsilon_{c}$ lies in

$$
0<\varepsilon_{c}<\varepsilon_{0} .
$$

As it can be seen from Eqs. (16)-(24), for the $\mathrm{TE}^{z}$ case, the transparency condition for the PEC cylinder is independent of the permeability of its metamaterial coating. As a matter of fact, this is true when the cylindrical scatterer is electrically small and the scattering problem is consequently "quasielectrostatic." Simply we will choose $\mu_{c}=\mu_{0}$ in the numerical experiments for convenience.

For a specific coating permittivity $\varepsilon_{c}$, utilizing Eq. (16), one can analytically find the core-coating ratio $\gamma$ at which transparency can be obtained. Similarly, one can rewrite Eq. (16) as

$$
\varepsilon_{c}=\frac{1-\gamma^{2 n}}{1+\gamma^{2 n}} \varepsilon_{0}
$$

to find the coating permittivity for a desired $\gamma$, again analytically.

Before providing any numerical results, it should be noted that $\varepsilon_{c}$ given in Eq. (25) depends on $n$. Therefore, one has to determine which $n$ value to use in Eq. (25). For this purpose, magnitudes of some scattering coefficients, $\left|c_{n}^{\mathrm{TE}}\right|$ versus $\gamma$, are plotted in Fig. 2 for a cylinder having outer radius $b$ $=\lambda_{0} / 100$. Our goal is to achieve transparency at $\gamma=0.5$. From Fig. 2 it is observed that $\left|c_{0}^{\mathrm{TE}}\right|$ increases with increasing $\gamma$. Since the outer radius " $b$ " is fixed, this means that $\left|c_{0}^{\mathrm{TE}}\right|$ increases when the inner radius " $a$ " is increased. It can be deduced that the scattering coefficient $c_{0}^{\mathrm{TE}}$ is mostly related to the PEC core cylinder and physically setting it to zero is not possible. Similarly, in [27] the $n=0$ term is shown to be equivalent to a $z$-directed magnetic line source. Magnitude of the next dominant term, $\left|c_{1}^{\mathrm{TE}}\right|(n= \pm 1)$ which are referred to as dipolar terms in [15], is also given in Fig. 2. As expected from Eq. (25), $\left|c_{1}^{\mathrm{TE}}\right|$ makes a dip at $\gamma=0.5$. For electrically 
TABLE II. Desired and obtained $\gamma$ for achieving transparency using Eq. (25).

\begin{tabular}{|c|c|c|c|c|c|c|c|c|}
\hline \multicolumn{3}{|c|}{$b=\lambda_{0} / 100$} & \multicolumn{3}{|c|}{$b=\lambda_{0} / 10$} & \multicolumn{3}{|c|}{$b=\lambda_{0} / 5$} \\
\hline Desired $\gamma$ & $\varepsilon_{c}$ & Obtained $\gamma$ & Desired $\gamma$ & $\varepsilon_{c}$ & Obtained $\gamma$ & Desired $\gamma$ & $\varepsilon_{c}$ & Obtained $\gamma$ \\
\hline 0.2 & $0.923 \varepsilon_{0}$ & 0.165 & 0.2 & $0.923 \varepsilon_{0}$ & 0.15 & 0.2 & $0.923 \varepsilon_{0}$ & 0.105 \\
\hline 0.5 & $0.6 \varepsilon_{0}$ & 0.41 & 0.5 & $0.6 \varepsilon_{0}$ & 0.39 & 0.5 & $0.6 \varepsilon_{0}$ & 0.31 \\
\hline 0.7 & $0.342 \varepsilon_{0}$ & 0.595 & 0.7 & $0.342 \varepsilon_{0}$ & 0.575 & 0.7 & $0.342 \varepsilon_{0}$ & 0.51 \\
\hline 0.9 & $0.105 \varepsilon_{0}$ & 0.81 & 0.9 & $0.105 \varepsilon_{0}$ & 0.805 & 0.9 & $0.105 \varepsilon_{0}$ & 0.78 \\
\hline
\end{tabular}

small cylinders, $c_{2}^{\mathrm{TE}}, c_{3}^{\mathrm{TE}}, \ldots$ are negligible. $\left|c_{2}^{\mathrm{TE}}\right|$ is shown in Fig. 2 for comparison with $\left|c_{0}^{\mathrm{TE}}\right|$ and $\left|c_{1}^{\mathrm{TE}}\right|$. Note that in accordance with Eq. (25), $\left|c_{2}^{\mathrm{TE}}\right|$ makes a dip at $\gamma=0.71$.

In Fig. 2, although $\left|c_{1}^{\mathrm{TE}}\right|$ is very close to zero when $\gamma$ $=0.5,\left|c_{0}^{\mathrm{TE}}\right|$ at the same $\gamma$ value is quite large. Considering only the three dominant scattering coefficients (i.e., $c_{0}^{\mathrm{TE}}$ and $c_{-1}^{\mathrm{TE}}=c_{1}^{\mathrm{TE}}$ ) for electrically small cylinders, for the monostatic case (i.e., $\phi-\phi_{0}=\pi$ ), the normalized monostatic echo width reduces to

$$
\sigma^{\mathrm{TE}} / \lambda_{0} \approx \frac{2}{\pi}\left|c_{0}^{\mathrm{TE}}-2 c_{1}^{\mathrm{TE}}\right|^{2}
$$

which tells us that the dipolar terms should be used to cancel the scattering from the PEC core (or $z$-directed magnetic line source) as oppose to conjugate pairing [9], which aims to make $c_{1}^{\mathrm{TE}}$ zero [see Eq. (25)]. In Fig. 2, $\left|c_{0}^{\mathrm{TE}}-2 c_{1}^{\mathrm{TE}}\right|$ shows a dip at $\gamma=0.41$, due to cancellation of $c_{0}^{\mathrm{TE}}$ with $c_{ \pm 1}^{\mathrm{TE}}$. Therefore, transparency is obtained at this $\gamma$ value, indeed. However, note that, as the electrical size of the cylindrical scatterer increases, higher order scattering coefficients (i.e., $c_{n}^{\mathrm{TE}}=c_{-n}^{\mathrm{TE}}$, $n \geqslant 2$ ) will become important and will degrade the approximation of Eq. (26). Consequently, the condition (25), which relates $\varepsilon_{c}$ to $\gamma$ (and which works fine for dielectric core cylinder cases [9]), should be modified.

To test the accuracy of Eq. (25) and to find (if possible) a better condition for transparency when the core cylinder is PEC, the following procedure is applied: For a desired $\gamma$ value, we analytically find what the coating permittivity, $\varepsilon_{c}$, should be. Then, using this coating permittivity, we numerically find at which $\gamma$ value transparency is actually obtained. In Table II, for certain outer shell radii some $\gamma$ values are selected where transparency is desired to be observed. The permittivities of the metamaterial coating corresponding to these $\gamma$ values after Eq. (25) [by setting $n=1$ in Eq. (25)] are tabulated in Table II. Based on numerical results, transpar- ency is obtained at different $\gamma$ values (reasonably below desired values), which are also tabulated in Table II. [Notice that when the core cylinder is replaced with a core-dielectric, $\varepsilon_{c}$ given in Eq. (17) yields accurate results as mentioned in [9] for electrically small cylinders.] It is also observed that as the electrical size of the cylindrical scatterer increases, deviation of the obtained $\gamma$ values from the the desired $\gamma$ values increases. This is an expected result since the accuracy of Eq. (25) decreases as the electrical size of the scatterer increases.

Based on Table II and our discussions on the scattering coefficients, it is noticed that the deviation between desired and obtained $\gamma$ values usually increases as the value of $\gamma$ increases. Therefore, we heuristically modify Eq. (25) as

$$
\varepsilon_{c}=\frac{1-\gamma^{(2 n-\gamma)}}{1+\gamma^{(2 n-\gamma)}} \varepsilon_{0},
$$

to find $\varepsilon_{c}$ for a desired $\gamma$ value, analytically. Note that, a theoretically more correct approach for finding the actual transparency condition is under study. Currently, a condition relating $\varepsilon_{c}$ to $\gamma$ by using $c_{0}^{\mathrm{TE}}-2 c_{1}^{\mathrm{TE}}=0$ in the subwavelength limit for electrically small cylinders, and a more general condition relating $\varepsilon_{c}$ to $\gamma$ by using $c_{0}^{\mathrm{TE}}-\sum_{n=1}^{N} 2 c_{n}^{\mathrm{TE}}=0$ are being investigated. However, the condition given in Eq. (27) yields very accurate results particularly for small cylinders.

Similar to Table II, desired $\gamma$ values, the corresponding $\varepsilon_{c}$ values and obtained $\gamma$ values where transparency occurs after Eq. (27) [again by setting $n=1$ ] are tabulated in Table III. As it can be seen from Table III, Eq. (27) decreases the deviation successfully, especially when $b \leqslant \lambda_{0} / 10$.

On the other hand, the transparency condition for the initial (conjugately paired) cylindrical structure for the $\mathrm{TM}^{z}$ polarization can be found from Eqs. (17) and (18) utilizing duality:

$$
\gamma=\sqrt[2 n]{\frac{\left(\mu_{c}-\mu_{0}\right)\left(\mu_{c}+\mu\right)}{\left(\mu_{c}-\mu\right)\left(\mu_{c}+\mu_{0}\right)}} \quad \text { for } n \neq 0,
$$

\begin{tabular}{|c|c|c|c|c|c|c|c|c|}
\hline \multicolumn{3}{|c|}{$b=\lambda_{0} / 100$} & \multicolumn{3}{|c|}{$b=\lambda_{0} / 10$} & \multicolumn{3}{|c|}{$b=\lambda_{0} / 5$} \\
\hline Desired $\gamma$ & $\varepsilon_{c}$ & Obtained $\gamma$ & Desired $\gamma$ & $\varepsilon_{c}$ & Obtained $\gamma$ & Desired $\gamma$ & $\varepsilon_{c}$ & Obtained $\gamma$ \\
\hline 0.2 & $0.895 \varepsilon_{0}$ & 0.19 & 0.2 & $0.895 \varepsilon_{0}$ & 0.175 & 0.2 & $0.895 \varepsilon_{0}$ & 0.125 \\
\hline 0.5 & $0.478 \varepsilon_{0}$ & 0.49 & 0.5 & $0.478 \varepsilon_{0}$ & 0.47 & 0.5 & $0.478 \varepsilon_{0}$ & 0.395 \\
\hline 0.7 & $0.228 \varepsilon_{0}$ & 0.68 & 0.7 & $0.228 \varepsilon_{0}$ & 0.67 & 0.7 & $0.228 \varepsilon_{0}$ & 0.625 \\
\hline 0.9 & $0.0579 \varepsilon_{0}$ & 0.875 & 0.9 & $0.0579 \varepsilon_{0}$ & 0.875 & 0.9 & $0.0579 \varepsilon_{0}$ & 0.86 \\
\hline
\end{tabular}

TABLE III. Desired and obtained $\gamma$ for achieving transparency using Eq. (27). 

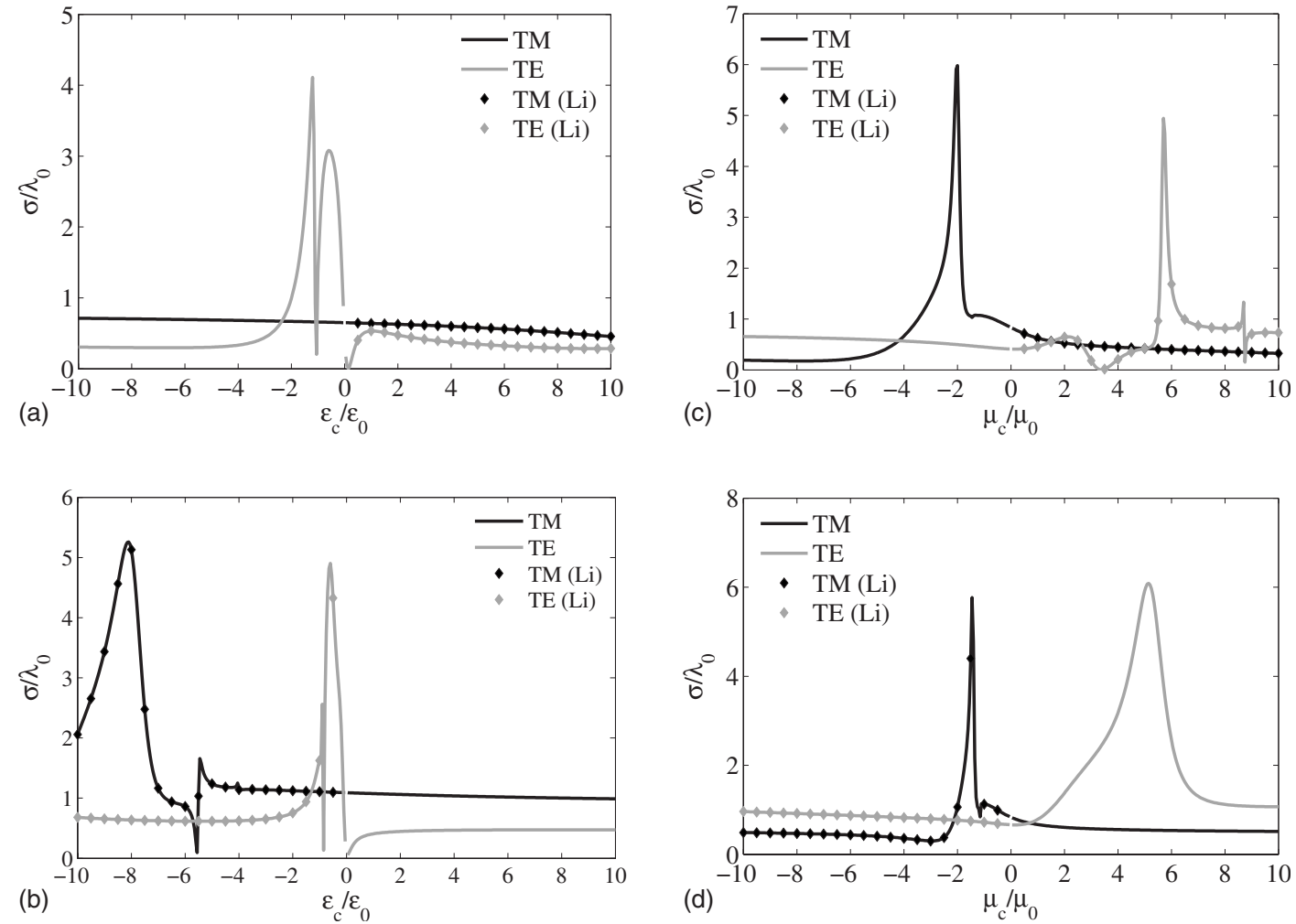

FIG. 3. Normalized monostatic echo width of a metamaterial-coated PEC cylinder $(a=50 \mathrm{~mm}, b=70 \mathrm{~mm}$, and $f=1 \mathrm{GHz})$. Diamond marks show the DPS and DNG coating cases in [8]. (a) $\mu_{c}=\mu_{0}$, (b) $\mu_{c}=-\mu_{0}$, (c) $\varepsilon_{c}=2.2 \varepsilon_{0}$, and (d) $\varepsilon_{c}=-2.2 \varepsilon_{0}$.

$$
\gamma=\sqrt{\frac{\varepsilon_{c}-\varepsilon_{0}}{\varepsilon_{c}-\varepsilon}} \text { for } n=0 .
$$

After replacing the core cylinder with a PEC one, Eqs. (28) and (29) become

$$
\begin{gathered}
\gamma=\sqrt[2 n]{\frac{\left(\mu_{c}-\mu_{0}\right)\left(\mu_{c}+\mu_{0}\right)}{\left(\mu_{c}-\mu_{0}\right)\left(\mu_{c}+\mu_{0}\right)}}=1 \text { for } \mu_{c} \neq \mu_{0}, \\
\mu_{c} \neq-\mu_{0}, \quad n \neq 0, \\
\gamma=\sqrt{\frac{\varepsilon_{c}-\varepsilon_{0}}{\varepsilon_{c}-\varepsilon}} \rightarrow \sqrt{\frac{\varepsilon_{c}-\varepsilon_{0}}{\varepsilon_{c}+j \infty}} \text { for } n=0 .
\end{gathered}
$$

It can be deduced from Eqs. (30) and (31) that the transparency condition for the $\mathrm{TM}^{z}$ polarization does not lead to any reasonable outcome due to the core being PEC. It is obvious that in DPS-DNG or ENG-MNG pairing no such difficulty arises since duality can be simply applied. To be able to achieve transparency for the $\mathrm{TM}^{z}$ polarization utilizing similar transparency conditions we have derived for $\mathrm{TE}^{z}$ polarization, the core should be PMC instead of PEC. Theoretical analysis or simply duality shows that in such a case one can use the dual of transparency condition for $\mathrm{TE}^{z}$ polarization by interchanging any permittivity with the corresponding permeability. Yet, even if the core cylinder is PEC, our numerical investigations show that transparency for $\mathrm{TM}^{z}$ polarization can be obtained for electrically small cylinders with metamaterial coatings having large $\left|\mu_{c}\right|$. Examples of this situation are illustrated in Sec. V (Numerical Results and Discussion).

\section{RESONANCE (SCATTERING MAXIMIZATION) CONDITION}

The resonance condition, which increases the scattering drastically for an electrically small cylindrical scatterer, is derived by setting the denominator of the scattering coefficient $c_{n}^{\mathrm{TE}}$ in Eq. (11) to zero, again in the sub-wavelength limit. This yields the following resonance condition:

$$
\gamma=\sqrt[2 n]{\frac{\varepsilon_{0}+\varepsilon_{c}}{\varepsilon_{0}-\varepsilon_{c}}} \text { for } n \neq 0
$$

Alternatively, one can use the resonance condition given in [12] for the $\mathrm{TE}^{z}$ polarization

$$
\gamma=\sqrt[2 n]{\frac{\left(\varepsilon_{c}+\varepsilon_{0}\right)\left(\varepsilon_{c}+\varepsilon\right)}{\left(\varepsilon_{c}-\varepsilon_{0}\right)\left(\varepsilon_{c}-\varepsilon\right)}} \quad \text { for } n>0
$$

When the core cylinder is PEC, Eq. (33) becomes

$$
\gamma \rightarrow \sqrt[2 n]{\frac{\left(\varepsilon_{c}+\varepsilon_{0}\right)\left(\varepsilon_{c}-j \infty\right)}{\left(\varepsilon_{c}-\varepsilon_{0}\right)\left(\varepsilon_{c}+j \infty\right)}}=\sqrt[2 n]{\frac{\varepsilon_{0}+\varepsilon_{c}}{\varepsilon_{0}-\varepsilon_{c}}} \text { for } n>0
$$

Since the root in Eq. (32) is of even degree of $n$ (i.e., $2 n$ ) and $0<\gamma<1$, then 

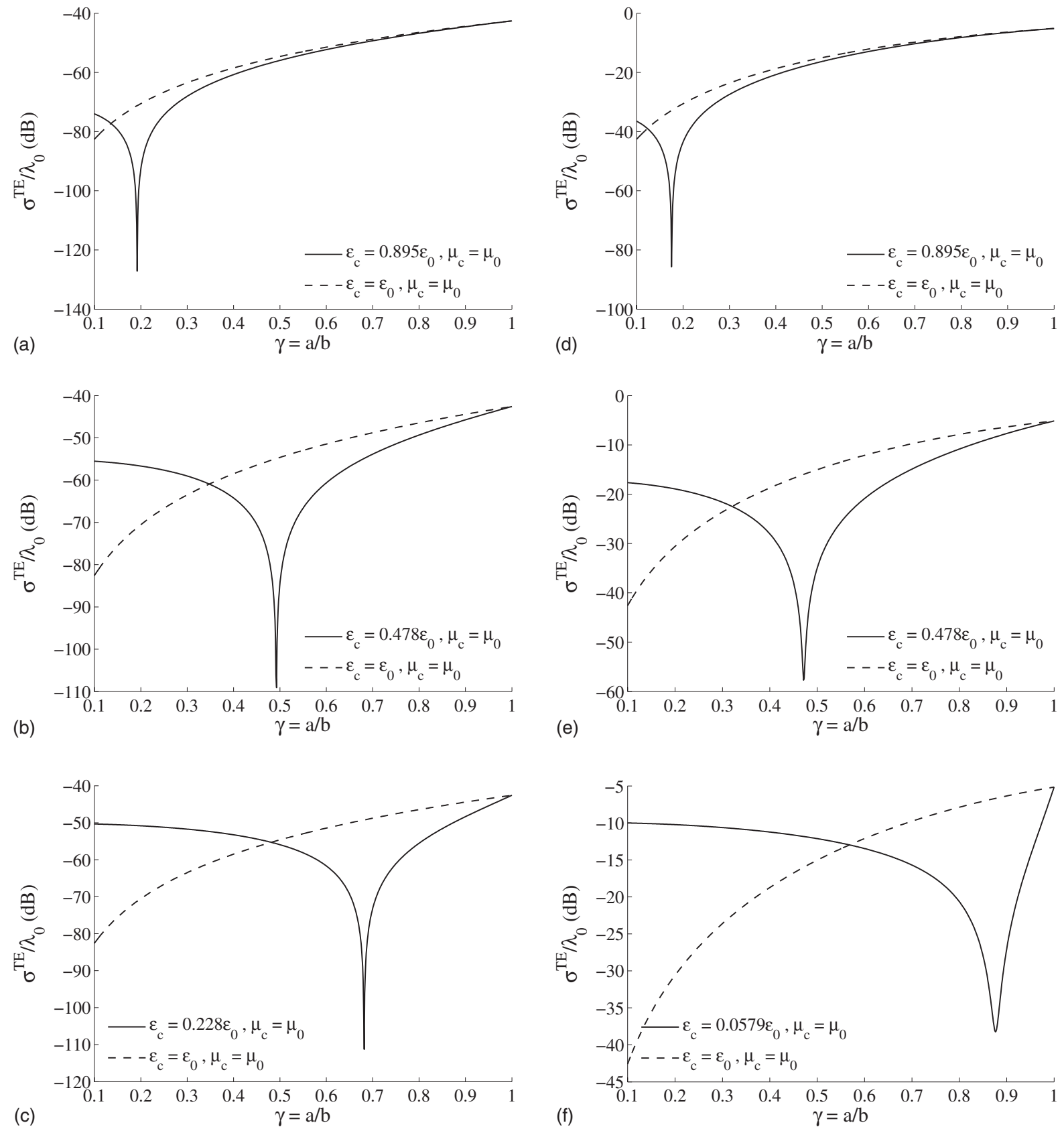

FIG. 4. Normalized monostatic echo width of a metamaterial-coated PEC cylinder for the TE ${ }^{z}$ polarization case vs the core-coating ratio for coatings with different constitutive parameters. The outer radius of the coating is selected as (a) - (c) $b=\lambda_{0} / 100$ and (d) $-(\mathrm{f}) b=\lambda_{0} / 10$. Dashed line shows the uncoated PEC case, with radius $a$.

$$
0<\frac{\varepsilon_{0}+\varepsilon_{c}}{\varepsilon_{0}-\varepsilon_{c}}<1
$$

which leads to

$$
0<\frac{\varepsilon_{0}+\varepsilon_{c}}{\varepsilon_{0}-\varepsilon_{c}} \Rightarrow-\varepsilon_{0}<\varepsilon_{c}<\varepsilon_{0}
$$

and

$$
\frac{\varepsilon_{0}+\varepsilon_{c}}{\varepsilon_{0}-\varepsilon_{c}}<1 \Rightarrow \varepsilon_{c}<0 \text { or } \varepsilon_{c}>\varepsilon_{0}
$$

From Eqs. (36) and (37), the proper choice for $\varepsilon_{c}$ lies in

$$
-\varepsilon_{0}<\varepsilon_{c}<0
$$

Then, the ratio of core-coating radii $\gamma$, to maximize scattering from a metamaterial-coated PEC cylinder, can be found analytically from the permittivity of the coating $\varepsilon_{c}$ utilizing Eq. (32), and vice versa: 

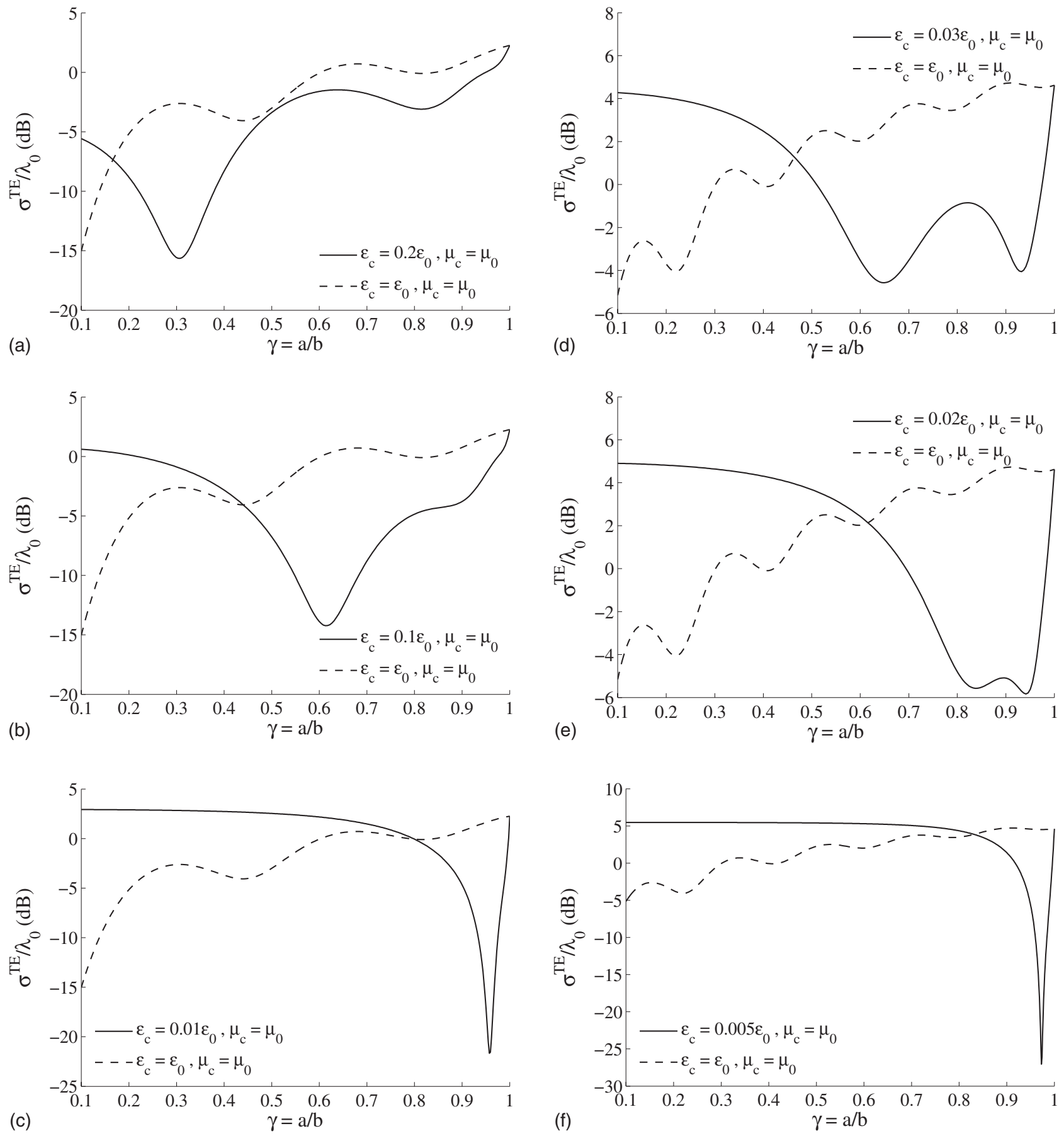

FIG. 5. Normalized monostatic echo width of a metamaterial-coated PEC cylinder for the TE ${ }^{z}$ polarization case vs the core-coating ratio for coatings with different constitutive parameters. The outer radius of the coating is selected as (a)-(c) $b=\lambda_{0} / 2$ and (d) $-(\mathrm{f}) b=\lambda_{0}$. Dashed line shows the uncoated PEC case, with radius $a$.

$$
\varepsilon_{c}=\frac{\gamma^{2 n}-1}{\gamma^{2 n}+1} \varepsilon_{0} .
$$

In our numerical experiments with scattering maximization, we follow the same procedure as in the transparency condition (i.e., we find the coating permittivity for a desired $\gamma$ value analytically and then use it in the numerical experiment). Our numerical experiments show that, for electrically small cylindrical scatterers, Eq. (39) works quite well (by setting $n=1)$. Therefore, we do not modify it as we have modified the analytical transparency relation.

To understand how this resonance condition occurs, consider a PEC cylinder which is illuminated by a $\mathrm{TE}^{z}$ polarized plane wave. As mentioned in the transparency phenomenon the $n=0$ term, that corresponds to the PEC case for a $z$-directed magnetic line source [27], becomes dominant when the cylinder is electrically very small. However, the $n= \pm 1$ terms (dipolar terms [15] that correspond to a 

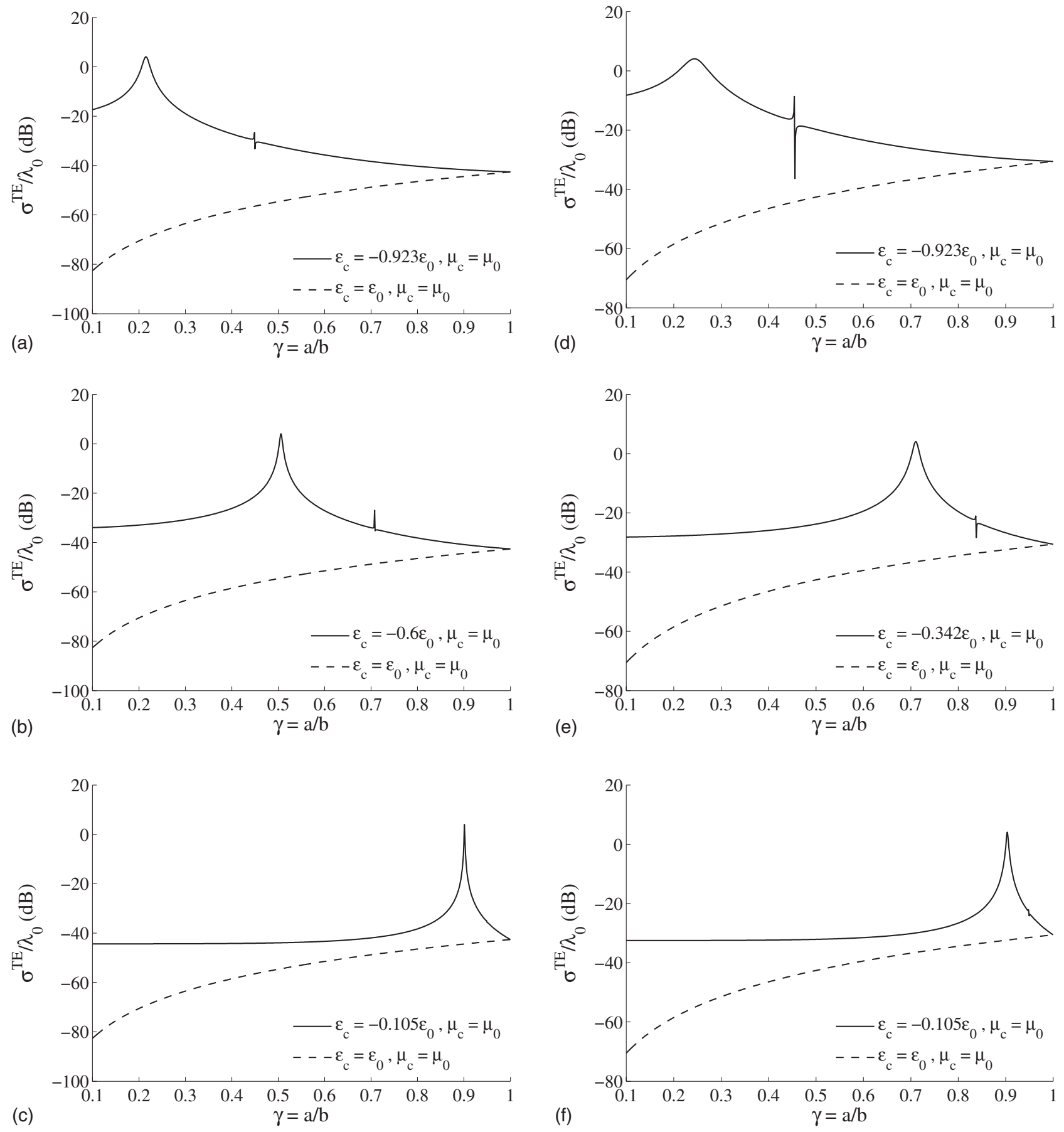

FIG. 6. Normalized monostatic echo width of an ENG coated PEC cylinder for the $\mathrm{TE}^{z}$ polarization case vs the core-coating ratio for coatings with different constitutive parameters. The outer radius of the coating is selected as (a)-(c) $b=\lambda_{0} / 100$ and (d) $-(\mathrm{f}) b=\lambda_{0} / 50$. Dashed line shows the uncoated PEC case, with radius $a$.

$y$-directed electric dipole) cannot be neglected since they radiate more efficiently [27]. Due to its electrically small size, this electric dipole behaves like a capacitive element. If there is also an ENG coating present, the coating will act like an inductive element. Therefore, the whole cylindrical scatterer will form an inductor-capacitor (LC) resonator. A similar scenario is investigated in [21] for electrically small antennas enclosed by metamaterial shells. As the size of the scatterer increases, quadrupolar (i.e., $n=2$ ), octopolar (i.e., $n=3$ ) and any higher order terms also emerge as resonant terms [15].
Interestingly, comparison of Eq. (25) with Eq. (39) for a desired $\gamma$ value shows that, the permittivity of the coating to maximize scattering should be the negative of the coating permittivity which makes the cylinder transparent. For the $\mathrm{TE}^{z}$ case, since the scattering maximization condition is independent of the permeability of its coating and for electrically small cylindrical scatterers we are dealing with the "quasielectrostatic" problem, we can safely choose $\mu_{c}=\mu_{0}$. Therefore, coatings we use here for scattering maximization are ENG metamaterials (or plasmonic materials). 

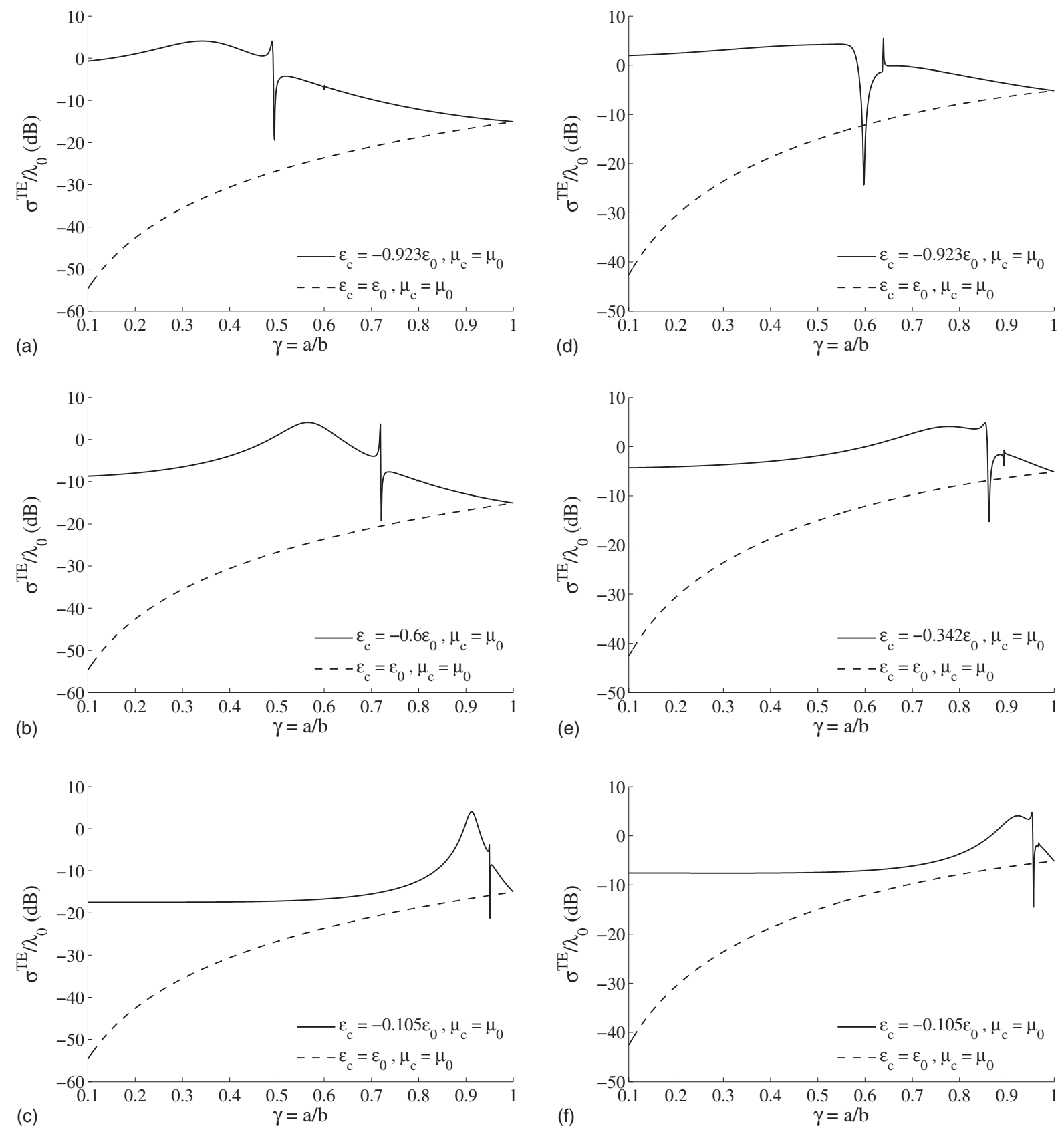

FIG. 7. Normalized monostatic echo width of an ENG coated PEC cylinder for the $\mathrm{TE}^{z}$ polarization case vs the core-coating ratio for coatings with different constitutive parameters. The outer radius of the coating is selected as (a) - (c) $b=\lambda_{0} / 20$ and (d) $-(\mathrm{f}) b=\lambda_{0} / 10$. Dashed line shows the uncoated PEC case, with radius $a$.

The resonance condition for the same cylindrical structure for the $\mathrm{TM}^{z}$ polarization, which can be derived from Eq. (33) utilizing duality, is given in [12] as

$$
\gamma=\sqrt[2 n]{\frac{\left(\mu_{c}+\mu_{0}\right)\left(\mu_{c}+\mu\right)}{\left(\mu_{c}-\mu_{0}\right)\left(\mu_{c}-\mu\right)}} \text { for } n>0 .
$$

After replacing the core cylinder with a PEC one, Eq. (40) becomes

$$
\gamma=\sqrt[n]{\frac{\mu_{c}+\mu_{0}}{\mu_{c}-\mu_{0}} \mid} \quad \text { for } \mu_{c} \neq \mu_{0}, \quad n>0 .
$$

Although Eq. (41) states a resonance relation between a desired $\gamma$ value and $\mu_{c}$ for the $\mathrm{TM}^{z}$ polarization, our numerical investigations show that $\mu_{c}$ values obtained via Eq. (41) (i.e., from the desired $\gamma$ values) yield resonance (i.e., maximum scattering) at $\gamma$ values different from the desired ones. On the other hand, similar to the transparency condition, if PEC core is replaced by a PMC core, then dual of Eq. (38) (i.e, $-\mu_{0}<\mu_{c}<0$ ) yields a resonance at the desired $\gamma$ value for the $\mathrm{TM}^{z}$ polarization.

Note that all the formulations used for transparency and scattering maximization conditions are independent of the electrical size of the cylindrical scatterer (i.e., $a$ and $b$ ). How- 

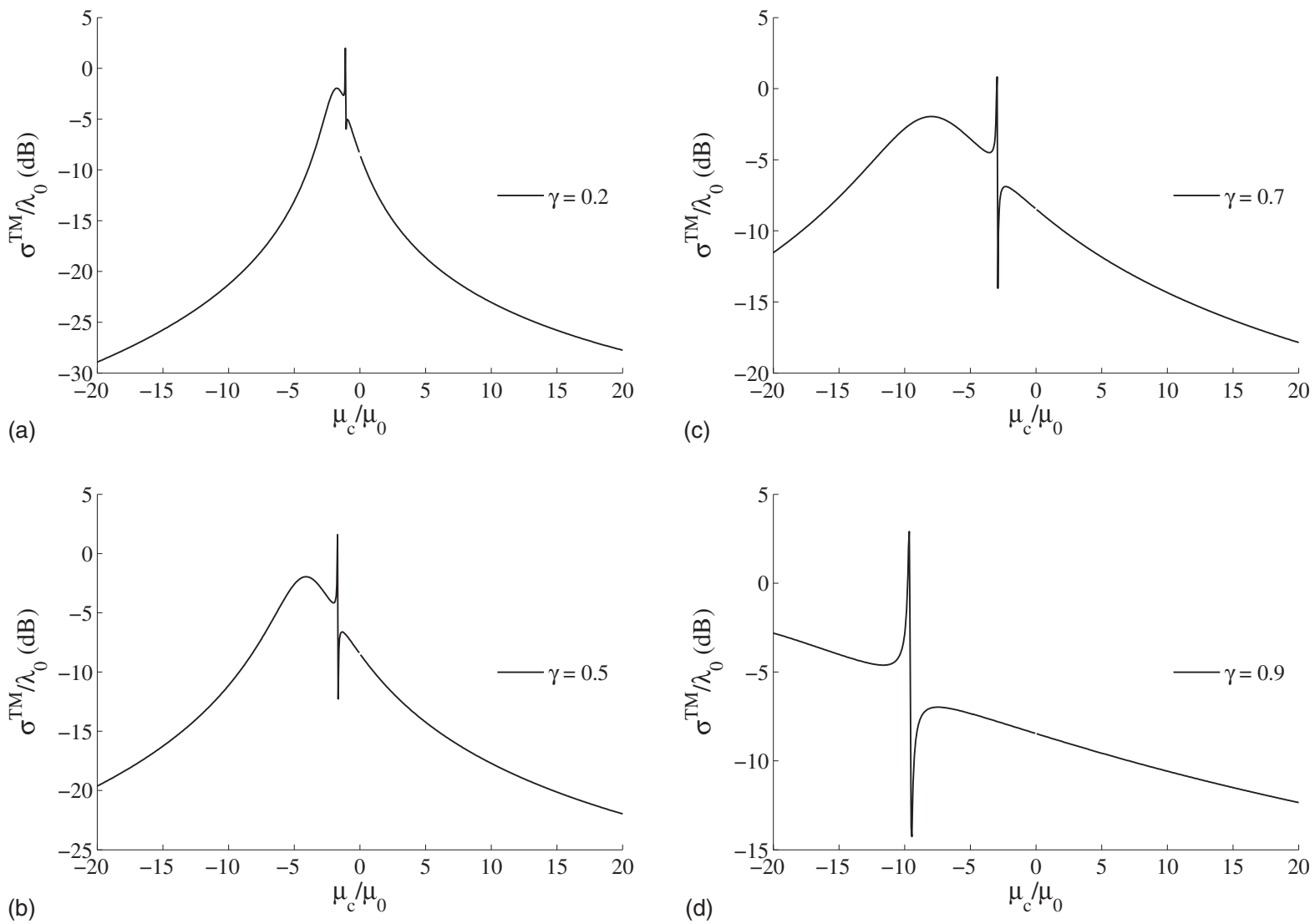

FIG. 8. Normalized monostatic echo width of a metamaterial-coated PEC cylinder for the $\mathrm{TM}^{z}$ polarization case vs the coating permeability $\mu_{c}$ for different core-coating ratios. The outer radius of the coating is $b=\lambda_{0} / 100$ and the coating permittivity is $\varepsilon_{c}=\varepsilon_{0}$.

ever, the formulations are expected to work well for electrically very small cylinders (i.e., $\left|k_{c}\right| b \ll 1, k_{0} b \ll 1$ ), such that only a few modes of the infinite series summation are enough to represent the whole radar cross section. Although the aforementioned theoretical analysis is based on electrically small cylinders, conditions relating $\varepsilon_{c}$ to $\gamma$ are found by setting $n=1$, and a few modes of the infinite series is assumed to be dominant, in the computation of the normalized echo widths we use sufficiently many modes to be accurate. In other words, our numerical results do not include any assumption in this sense.

\section{NUMERICAL RESULTS AND DISCUSSION}

To assess the accuracy of our numerical routines, we have duplicated one of the numerical results (normalized monostatic echo width of a metamaterial coated PEC cylinder at $1 \mathrm{GHz}$ with PEC radius $a=50 \mathrm{~mm}$ and coating radius $b$ $=70 \mathrm{~mm}$ ) in [8], which is shown in Fig. 3. In addition to the DPS and DNG coatings investigated in [8], we also included ENG and MNG coatings. As seen in Fig. 3, we have good agreement with the results of [8]. Moreover, a continuation in the monostatic echo width values is observed (as expected) when the coating medium becomes single-negative (SNG) from a DPS or DNG coating.

In the previous sections, expanding the transparency condition given in [9], we have found that it is possible to make PEC cylinders transparent for the $\mathrm{TE}^{z}$ polarization by cover- ing them with metamaterial covers which exhibit the material property given by Eq. (24). By transparency we mean the significant reduction and minimization of scattering in the backscattering direction. As it has been explained previously, the transparency condition is expected to work well for electrically very small cylinders. Therefore, we start with an electrically very small PEC cylinder (in the cross-sectional sense) covered with our proposed metamaterial coating such that the outer radius of the coating is $b=\lambda_{0} / 100$. Then, for some $\gamma$ values, where transparency is desired to be observed, the corresponding permittivities are analytically found using Eq. (27) as tabulated in Table III. Finally, the normalized monostatic echo widths are calculated and depicted in Figs. 4(a)-4(c) for these permittivities. One can see that transparency is indeed obtained for PEC cylinders almost at the desired $\gamma$ values. Note that the dashed lines indicate the normalized monostatic echo widths for uncoated PEC cylinders (i.e., with radius $a=\gamma . b$ ) such that the metamaterial coating (i.e., the region $a \leqslant \rho \leqslant b$ ) is replaced by free space. As seen in all figures, at the desired $\gamma$ value, the reduction in backscattering is significant when proposed metamaterial coatings are used. Note that for the uncoated case small $\gamma$ values mean extremely small PEC cylinders and as " $a$ " goes to zero no scattering is supposed to take place.

As the next step, we investigate what happens to the transparency as the electrical size of the scatterer increases. For this purpose, we gradually increase the outer radius of the cylindrical scatterer. The normalized monostatic echo 

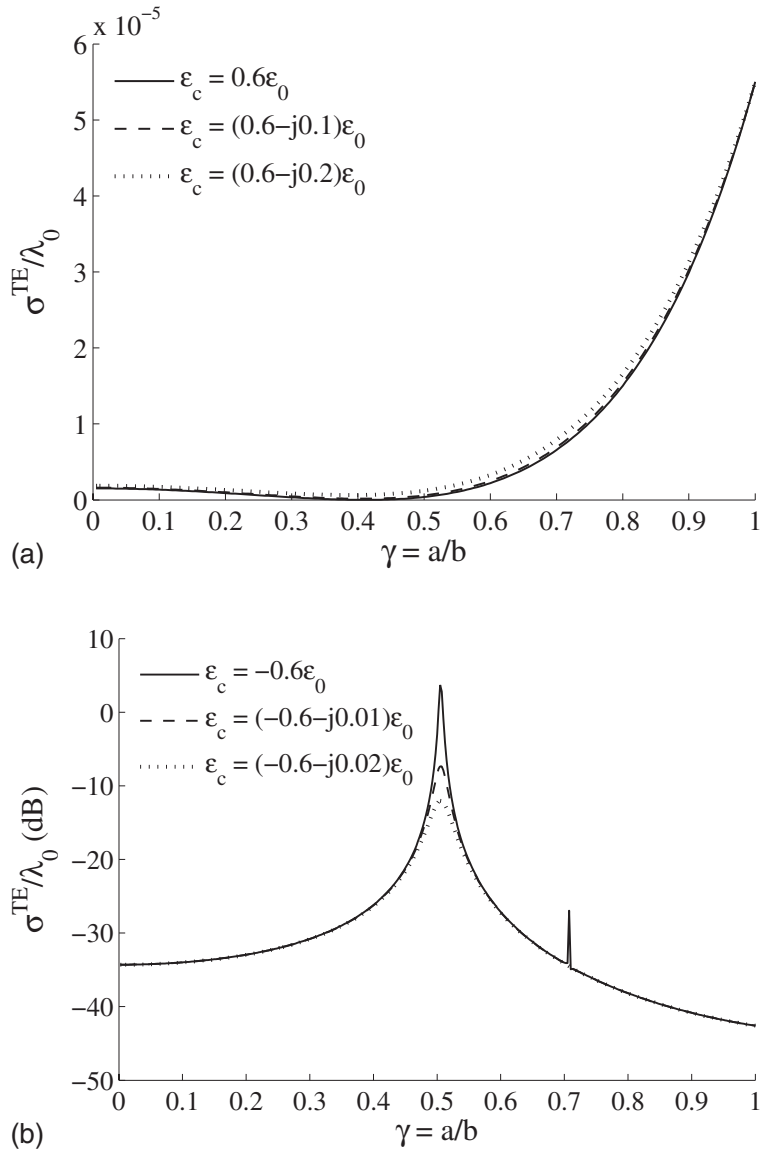

FIG. 9. Effects of ohmic losses on normalized monostatic echo width for (a) DPS (transparency) and (b) ENG (scattering maximization) cases. The outer radius of the coating is selected as $b$ $=\lambda_{0} / 100$.

widths are calculated and depicted in Figs. 4(d)-4(f), when the outer radius of the scatterer is increased to $b=\lambda_{0} / 10$. From Figs. 4(d)-4(f) we see that increasing the electrical size of the cylindrical scatterer from $b=\lambda_{0} / 100$ to $b=\lambda_{0} / 10$ increases the RCS considerably (e.g., the largest normalized monostatic echo width increases roughly from $-40 \mathrm{~dB}$ to $-5 \mathrm{~dB}$ ). Despite this huge increase in RCS, as it can be seen from Figs. 4(d)-4(f) and Table III, transparency can be achieved at the desired $\gamma$ values. Similarly, we can still achieve transparency close to desired $\gamma$ values (as tabulated in Table III) when the outer radius of the scatterer is increased to $b=\lambda_{0} / 5$.

Figure 4 and Table III show that as the permittivity of the coating is decreased from $\varepsilon_{c}=\varepsilon_{0}$ to $\varepsilon_{c}=0$, the core-coating ratio where transparency occurs moves from $\gamma=0$ to $\gamma=1$. To explain this phenomenon, we can treat the metamaterial coating as a cover which cancels out the electromagnetic response of the PEC core. When the permittivity of the metamaterial coating is close to $\varepsilon_{0}$, this cancellation is quite weak (i.e., metamaterial cover behaves like free space). In this case, the PEC core should be considerably small with respect to the coating such that a full cancellation can occur. However, when the permittivity of the coating is decreased towards 0 , the cancellation of the coating will become stronger, which means that with even thinner coatings it becomes
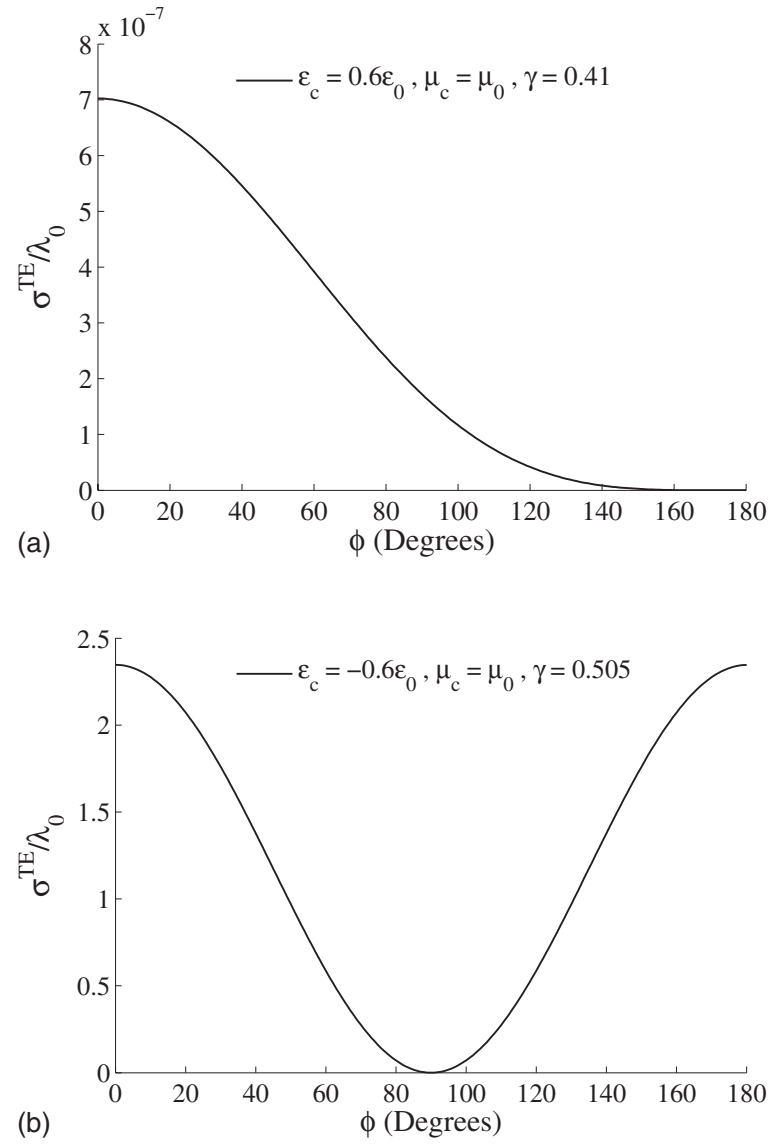

FIG. 10. Normalized bistatic echo widths for (a) DPS coated and (b) ENG coated PEC cylinder for the $\mathrm{TE}^{z}$ polarization case. The outer radius of the coating is selected as $b=\lambda_{0} / 100$. The angle of incidence is $\phi_{0}=0^{\circ}$.

possible to make larger PEC cores transparent. Note that a similar discussion is made in [9] to explain the cancellation phenomenon for metamaterial coated dielectric spheres. For both the dielectric core and the metamaterial cover, their polarization vectors are defined, respectively as $\mathbf{P}=\left(\varepsilon-\varepsilon_{0}\right) \mathbf{E}$ and $\mathbf{P}_{\mathbf{c}}=\left(\varepsilon_{c}-\varepsilon_{0}\right) \mathbf{E}$. The transparency condition is attributed to the cancellation of these antiparallel polarization vectors, which happens when $\varepsilon_{c}<\varepsilon_{0}$. In our scenario, since the core cylinder is PEC, the problem has a less degree of freedom and the analytical solution shows that to achieve transparency $0<\varepsilon_{c}<\varepsilon_{0}$ should be.

To see the limitations on the electrical size of the cylindrical scatterers for achieving transparency, we will consider relatively larger scatterers. Since these scatterers are electrically large, available analytical relations between $\gamma$ and $\varepsilon_{c}$ do not hold any longer. Therefore, for these large scatterers we choose $\varepsilon_{c}$ in a trial and error process. Figures 5(a)-5(c) show the results when the outer radius of the scatterer is increased to $b=\lambda_{0} / 2$. In Figs. 5(d)-5(f) this outer radius is further increased to $b=\lambda_{0}$. As it is seen in Fig. 5(a) and Fig. 5(d), the normalized monostatic echo width makes two dips at some $\gamma$. As the permittivity of the coating is decreased towards 0 , the dips move towards $\gamma=1$, destructively interfering with each other. Finally, the minimum value of the normalized echo width $\left(\sigma^{\mathrm{TE}} / \lambda_{0}\right.$ drops from $4 \mathrm{~dB}$ to $\left.-25 \mathrm{~dB}\right)$ is achieved 

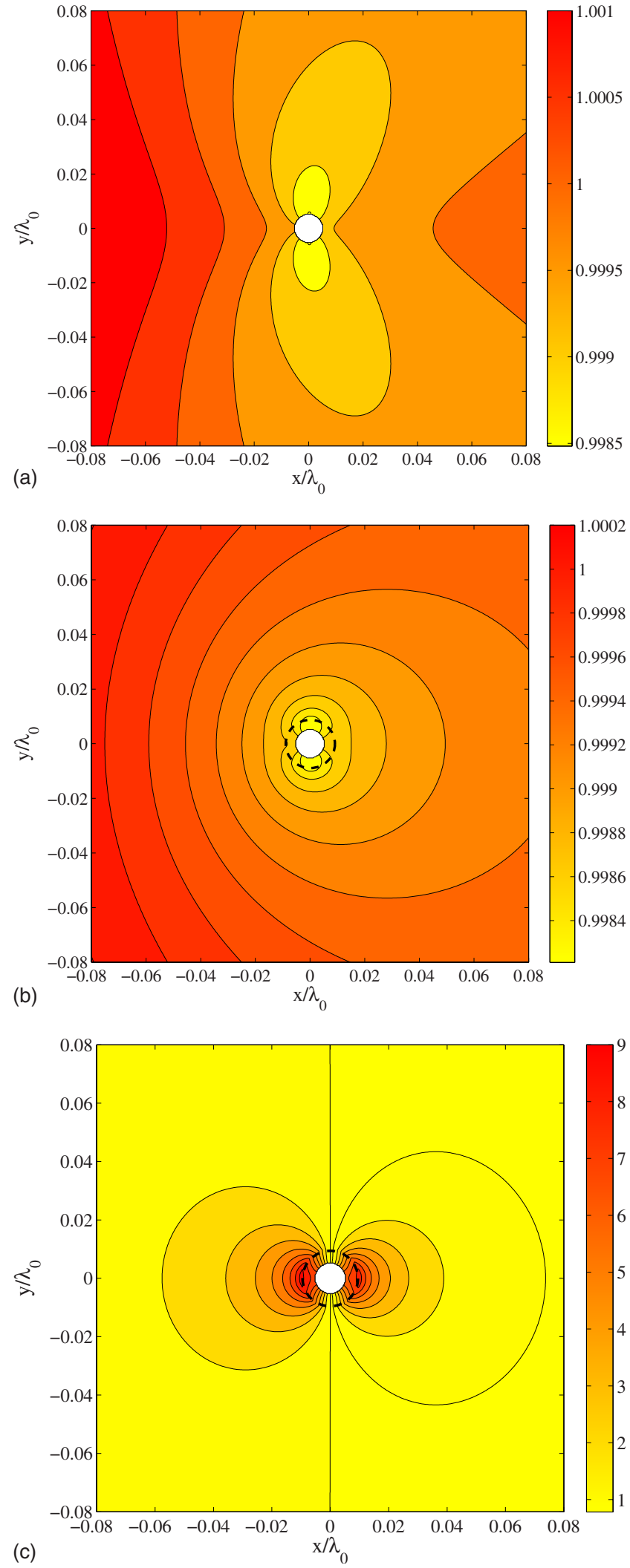

FIG. 11. (Color online) Contour plots of axial component of the total magnetic field (i.e., $H_{z}^{i}+H_{z}^{s}$ ) outside the PEC cylinder when there is (a) no coating, (b) DPS coating $\left(\varepsilon_{c}=0.6 \varepsilon_{0}, \mu_{c}=\mu_{0}\right)$, and (c) ENG coating $\left(\varepsilon_{c}=-0.6 \varepsilon_{0}\right.$ and $\left.\mu_{c}=\mu_{0}\right)$. Outer boundaries of the coatings are shown by dashed lines $\left(a=\lambda_{0} / 200\right.$ and $\left.b=\lambda_{0} / 100\right)$. Plane wave illumination is along the $+x$ axis. when the permittivity is very close to zero but positive, and $\gamma$ being between 0.9 and 1 . Therefore, larger cylinders require coatings having permittivities much closer to zero. Since monostatic echo width is minimized in the $0.9<\gamma<1$ region, the PEC core can be quite large.

Next, we turn our attention to investigate the validity of scattering maximization condition. Hence, we follow a procedure similar to the one we have done for the transparency condition. We again start with electrically very small cylindrical scatterers and gradually increase their outer radii. We use the same $\gamma$ in Table II as our desired $\gamma$ values, but this time to maximize scattering. Hence the coating permittivities are the negatives of coating permittivities tabulated in Table II, as a result of Eq. (39). Figures 6(a)-6(c) show the normalized monostatic echo widths for ENG coated PEC cylinders when the outer radius of the scatterer is $b=\lambda_{0} / 100$. As it can be seen from the figures, RCS increases drastically at the desired $\gamma$ values, making peaks, depending on the permittivity of the coating. This is mainly due to the resonance of dipolar terms which we have explained previously. When the outer radius is $b=\lambda_{0} / 50$, the RCS peaks can still be clearly seen in Figs. 6(d)-6(f). But, this time the peaks are wider and the peak centers deviate a little from their desired locations. Also note a second small peak which just emerges in Fig. $6(d)$ due to the quadrupolar terms. These quadrupolar terms become more observable in Figs. 7(a)-7(c) where $b=\lambda_{0} / 20$. When the outer radius is increased to $b=\lambda_{0} / 10$, effects of other higher order terms can be observed from Figs. 7(d)-7(f). In summary, Figs. 6 and 7 suggest that as the electrical size of the scatterer increases the peak due to the dipolar term becomes wider and moves towards $\gamma=1$. Also, due to the increased size, quadrupolar and higher order modes emerge. However, the peak due to the dipolar term is much more dominant and can be safely used to maximize RCS of objects.

To see whether any transparency or scattering maximization condition can be obtained for the $\mathrm{TM}^{z}$ polarization, we consider an electrically very small cylindrical scatterer with outer radius $b=\lambda_{0} / 100$. For various $\gamma$ values, we calculate the monostatic echo widths when $\mu_{c} / \mu_{0}$ is in the [-20 20] interval, as shown in Fig. 8. For this "quasimagnetostatic" problem, we have chosen $\varepsilon_{c}=\varepsilon_{0}$ for convenience. In Fig. 8, the double peaks oriented up and down are due to the resonance in $c_{ \pm 1}^{\mathrm{TM}}$ terms and these resonant modes maximize the RCS considerably, when $\mu_{c}<0$. Transparency can be obtained with coatings having large permeabilities in the absolute sense as seen in Figs. 8(a)-8(c). For $\gamma=0.9$, transparency is possible if $\mu_{c}$ is positive and very large.

As we have mentioned previously, the huge increase in the RCS of an ENG coated PEC cylinder is due to high resonance. However, transparency we have achieved using DPS coatings is not a result of such resonance, but simple cancellation. This can be best observed from the changes in RCS with respect to $\gamma$, when Figs. 4 and 5 are plotted in linear scale. In this case, it can be seen that RCS is not very sensitive to $\gamma$ near the transparency point. On the contrary, in Fig. 6 we see high $\gamma$ sensitivity. Since transparency condition is not a result of resonation, we also expect it not to be very sensitive to ohmic losses. For the ENG coated cases, however, there would be high sensitivity to ohmic losses near the 
resonant modes. The effects of small ohmic losses, as in the Drude or Lorentz medium models, are shown in Fig. 9. As predicted, there is very little ohmic sensitivity for transparency condition in Fig. 9(a). On the other hand, the high sensitivity to ohmic losses can be seen clearly at the resonance location in Fig. 9(b). Again in Fig. 9(b), despite the decrease in the monostatic echo width due to the ohmic losses, metamaterial coating provides at least approximately $65 \mathrm{~dB}$ increase in the echo width at the resonance location, when compared with the uncoated case.

In the numerical results we have shown up to here, we have considered the normalized monostatic echo widths (i.e., back scattering). To visualize the far-zone field distribution in the $x y$ plane, bistatic echo widths can be calculated. Figure 10 illustrates the bistatic scattering scenarios for transparency and scattering maximization for the TE polarization considering a metamaterial coated PEC cylinder with $b$ $=\lambda_{0} / 100$. The angle of incidence is set to $\phi_{0}=0^{\circ}$. In Fig. 10 (a), for the values of $\varepsilon_{c}=0.6 \varepsilon_{0}, \mu_{c}=\mu_{0}$ and $\gamma=0.41$, it is seen that RCS increases gradually from backscattering direction $\left(\phi=180^{\circ}\right)$ towards direction of incidence $\left(\phi=0^{\circ}\right)$. Therefore, while little portion of the incident wave is reflected back, the much larger portion will continue traveling in the direction of incidence. Indeed, this is the expected situation for transparency. In Fig. 10(b), for $\varepsilon_{c}=-0.6 \varepsilon_{0}, \mu_{c}$ $=\mu_{0}$, and $\gamma=0.505, \mathrm{RCS}$ is maximized in the backscattering and incidence directions, however it reduces towards $\phi$ $=90^{\circ}$, finally becoming effectively zero in this direction. In other words, RCS is not only maximized in the backscattering direction, but also in the direction of incidence.

Figure 11(a) shows the contour plot of the axial component of the total magnetic field (i.e., $H_{z}^{i}+H_{z}^{s}$ ) in the presence of single PEC cylinder, with radius $a=\lambda_{0} / 200$. In Fig. 11(b), the PEC cylinder is coated with a DPS metamaterial coating having $b=\lambda_{0} / 100, \varepsilon_{c}=0.6 \varepsilon_{0}$ and $\mu_{c}=\mu_{0}$. Comparison of Figs. 11(a) and 11(b) shows the decrease in RCS with the proposed metamaterial coating, especially in the backscattering direction. The case for the resonant ENG coating, for $b$ $=\lambda_{0} / 100, \varepsilon_{c}=-0.6 \varepsilon_{0}$, and $\mu_{c}=\mu_{0}$, which increases the RCS dramatically, is shown in Fig. 11(c). The field distribution confirms the strong resonance in the radiation of a $y$-directed electric dipole.

\section{CONCLUSION}

In this work, metamaterial coated PEC cylinders are investigated for achieving transparency and maximizing scattering. These infinitely long cylindrical scatterers are normally illuminated with monochromatic plane waves. The electromagnetic scattering problem is solved for the decoupled $\mathrm{TM}^{z}$ and $\mathrm{TE}^{z}$ polarizations separately. A general solution is obtained for DPS, ENG, MNG, and DNG metamaterial coatings which are homogeneous, isotropic, and linear, thus simple, and can be lossless or can have small electric or magnetic losses.

It is found out that rigorous derivation of transparency condition for PEC core case under the subwavelength limitations yields a similar transparency condition to that of two electrically small concentric layers of conjugately paired cyl- inders. Hence we demonstrate that transparency can indeed be achieved for metamaterial coated PEC cylinders. This transparency condition, which is found to be valid for $\mathrm{TE}^{z}$ polarization, requires a metamaterial coating having $0<\varepsilon_{c}$ $<\varepsilon_{0}$. However, the available relation between the permittivity of the coating, $\varepsilon_{c}$, and the ratio of core-coating radii, $\gamma$, becomes less accurate when the core dielectric cylinder is replaced by a PEC one. Therefore, the relation between $\gamma$ and $\varepsilon_{c}$ is modified in a sense that scattering from the PEC core is canceled with the dipolar terms. It has been shown that significant RCS minimization can be achieved even with large cylinders having outer radius $b=\lambda_{0}$. However, as the electrical size of the cylinder increases, the DPS coating should be thinner and it should have a permittivity much closer to zero. For larger cylinders, $\varepsilon_{c}$ and $\gamma$ cannot be related to each other with simple analytical or heuristic formulas.

In a similar fashion, for scattering maximization, we extended the resonance condition of two electrically small concentric layers of conjugately paired cylinders to metamaterial-coated PEC cylinders. Interestingly, rigorous derivation of the resonance condition yields similar results to that for conjugately paired cylinders (in the PEC core limit case) including the relation between $\gamma$ and $\varepsilon_{c}$. Substitution of the core cylinder with PEC shows that for $\mathrm{TE}^{z}$ polarization RCS of the cylindrical scatterer can be increased drastically, even when its electrical size is very small, using metamaterial or plasmonic covers having $-\varepsilon_{0}<\varepsilon_{c}<0$. The resonance peaks are due to resonant modes and the most dominant mode is the dipolar mode. As the size of the cylinder increases other higher order modes also emerge.

For $\mathrm{TM}^{z}$ polarization, even though there is no successful analytical relation for transparency or scattering maximization, numerical results show that for electrically small cylinders transparency can be obtained with metamaterial coatings having large permeabilities in the absolute sense and scattering maximization can be ensured since there exist resonant peaks when the coating permeability is less than zero. For $\mathrm{TM}^{z}$ polarization, the duals of transparency and scattering maximization conditions can also be obtained if the PEC core is replaced by a PMC core.

Effects of ohmic losses have also been investigated. As it is expected, transparency condition is not very sensitive to ohmic losses since it is not based on any resonance. However, scattering maximization is very sensitive to ohmic losses due to such resonances. Although ohmic losses of the metamaterial coating degrade the maximization in scattering, when compared with the lossless cases, the increase in the RCS remains successfully large, compared with the uncoated cases.

Numerical results for the bistatic RCS show that, in the transparency condition, little portion of the incident wave is reflected back and most portion continues traveling in the direction of incidence. Therefore, the transparency condition is satisfied, as intended. For scattering maximization, it is noticed that scattering can be maximized not only in the backscattering direction but also in the direction of incidence. Finally, the near-field contour plots, which visualize the axial component of the total magnetic field, show the decrease in the field intensities for transparency condition 
and the existence of a resonant $y$-directed electric dipole for scattering maximization. Our efforts on this topic are now concentrated on (i) finding a more general transparency condition to relate $\varepsilon_{c}$ to $\gamma$ that will work for large PEC objects, (ii) improving the transparency and scattering maximization conditions for $\mathrm{TM}^{z}$ polarization case by a thorough analytical formulation, (iii) investigation of oblique incidence problems, and (iv) at last but not the least, considerations of finite cylinders.

\section{ACKNOWLEDGMENTS}

This work was supported by the Turkish Scientific and Technical Research Agency (TÜBİTAK) under Grants No. EEEAG-104E044 and No. EEEAG-105E065 and by the Turkish Academy of Sciences (TÜBA)-GEBIP. The authors would like to thank Ekmel Özbay and anonymous referees for their useful discussions and comments.
[1] L. I. Mandelshtam, Lectures on Some Problems of the Theory of Oscillations (1944), Complete Collection of Works, Vol. 5 (Academy of Sciences, Moscow, 1950), pp. 428-467 (in Russian).

[2] L. I. Mandelshtam, Zh. Eksp. Teor. Fiz. 15, 476 (1945).

[3] V. G. Veselago, Sov. Phys. Usp. 10, 509 (1968).

[4] D. R. Smith, W. J. Padilla, D. C. Vier, S. C. Nemat-Nasser, and S. Schultz, Phys. Rev. Lett. 84, 4184 (2000).

[5] X. Zhou and G. Hu, Phys. Rev. E 74, 026607 (2006).

[6] J. B. Pendry, D. Schurig, and D. R. Smith, Science 312, 1780 (2006).

[7] S. A. Cummer, B. I. Popa, D. Schurig, D. R. Smith, and J. Pendry, Phys. Rev. E 74, 036621 (2006).

[8] C. Li and Z. Shen, Prog. Electromagn. Res. 42, 91 (2003).

[9] A. Alù and N. Engheta, Phys. Rev. E 72, 016623 (2005).

[10] A. Alù and N. Engheta, IEEE Trans. Antennas Propag. 51, 2558 (2003).

[11] A. Alù and N. Engheta, Opt. Express 15, 3318 (2007).

[12] A. Alù and N. Engheta, in Proceedings of the International Conference on Electromagnetics in Advanced Applications (Torino, Italy, 2003), pp. 435-438.

[13] A. Alù and N. Engheta, Progress in Electromagnetic Research Symposium (Waikiki, HI, 2003), p. 12.

[14] A. Alù and N. Engheta, in Proceedings of the 2004 IEEE Radar Conference (Philadelphia, PA, USA, 2004).
[15] A. Alù and N. Engheta, J. Appl. Phys. 97, 094310 (2005).

[16] A. Alù, N. Engheta, and R. W. Ziolkowski, in USNC-URSI Nat. Radio Science Meeting Dig. (Monterey, CA, 2004), p. 18.

[17] S. Arslanagic and O. Breinbjerg, IEEE Antennas Propag. Mag. 48, 38 (2006).

[18] S. Arslanagic, R. W. Ziolkowski, and O. Breinbjerg, Microwave Opt. Technol. Lett. 48, 2598 (2006).

[19] R. W. Ziolkowski and A. D. Kipple, IEEE Trans. Antennas Propag. 51, 2626 (2003).

[20] A. Erentok and R. W. Ziolkowski, in International Workshop on Antenna Technology: Small Antennas and Novel Metamaterials (White Plains, New York, 2006), pp. 400-403.

[21] R. W. Ziolkowski and A. Erentok, IEEE Trans. Antennas Propag. 54, 2113 (2006).

[22] W. L. Barnes, A. Dereux, and T. W. Ebbesen, Nature (London) 424, 824 (2003).

[23] C. A. Balanis, Advanced Engineering Electromagnetics (Wiley, New York, 1989), Chap. 11, pp. 595-596.

[24] D. R. Smith and N. Kroll, Phys. Rev. Lett. 85, 2933 (2000).

[25] J. B. Pendry, Phys. Rev. Lett. 85, 3966 (2000).

[26] R. W. Ziolkowski and E. Heyman, Phys. Rev. E 64, 056625 (2001).

[27] R. F. Harrington, Time-Harmonic Electromagnetic Fields (McGraw-Hill, New York, 1961), Chap. 5, pp. 235-236. 